\title{
How and why of vesicle formation
}

\section{Much has been done in the past few years to throw light on the energetics of vesicle formation in monolayer and bilayer membranes. The physics of the process is becoming clear, but the chemistry remains obscure.}

THE justifiable complaint that mainstream molecular biology is too neglectful of the quantitative side of the scientific process cannot, mercifully, be levelled at the whole of biology. On the contrary, there are some niches in which the application of techniques familiar in physics to problems in biology is well on the way to being a growth industry. One of the more spectacular of these is the study of the budding of vesicles from lipid membranes, which can be traced back at least as far as an article by P.B.Canham in 1970 (J. Theor. Biol. 26, 61; $1970)$; the objective, then, was simply to show that if, for example, a closed membrane forms a vesicle-shaped bud, the explanation must be that the budded form has less free energy than the unbudded form.

Nobody will dispute the importance of the process of vesicle formation. Cells that secrete enzymes or hormones do so by means of vesicles budding from their surfaces. Neurotransmitters are handled in the same way, as is the transport of cellular components within a cell, between the successive layers in the Golgi apparatus, for example. Replicated virus particles similarly emerge from infected cells by a budding process, while the inverse of that process, endocytosis, is the way in which cells ingest all but the simplest molecular structures from the outside. And what is to be made of the humble red blood cell, which is simply a free-standing membrane filled with haemoglobin whose normal shape is that of a doughnut or bagel?

And on the face of things, the budding of vesicles from the surface of a cell should be an easy problem. Just think of the outer membrane as a simple homogenous bipolar monolayer and suppose that the departure of the membrane from perfect flatness entails an energy cost - a 'bending energy' whereupon the energy of the membrane will be simply a function of the bending energy and the curvature over the surface. (The analogy with the stability of a drop of, say, water is not exact.)

In practice, there are complications. The strict analogue of surface tension is the relatively smaller force that holds a membrane laterally together. The bending energy presumably arises differently, by forcing on the molecules that constitute the membrane an orientation different from that in a flat membrane, perhaps bringing similarly charged groups closer together. Moreover, there is no reason why the forces in such a membrane should not be asymmetrical; bending in one direction may require more energy than bending by the same amount in the other. Symmetrical bilayers will naturally be different.

In the end, the calculation of the shape of a cell, and by extension of the vesicles that bud from it, is a matter of finding the closed surface whose energy is microscopically defined by its radii of curvature (in principle, there are two) at every point and two parameters representing the bending energy. All this has been elegantly explained in a review by Reinhard Lipowsky (Nature 349, 475-481; 1991).

Among other things, this argument explains why vesicle formation is such a common part of life. Start with a flat membrane and there are two components of the energy: the bending energy, zero for a symmetrical membrane, and the peripheral energy arising from the interaction of the edge of the membrane with the outside medium. That energy must be positive, perhaps because of hydrophobic interactions, or the membrane would not have formed in the first place. But the bending energy of a spherical closed membrane turns out not to depend on the radius of the sphere that it forms, but only on the numerical values of the two bending energy parameters.

So if the dimensions of the membrane are sufficiently large, it must be energetically favourable that the membrane should wrap up into a sphere. But, 'other things being equal', the energy embodied in a large sphere should be less than that of two smaller spheres of the same surface area. Endocytosis should be favoured, the budding of vesicles not. But that is not what real life shows, not to mention the increasingly elegant experiments with synthetic membranes that people have devised.

What can be the explanation? That 'other things' are not equal, of course. Predictably, the burgeoning theory of the membranebudding industry is busily relaxing the assumptions underlying the simple calculations. There are several variables to play with. Experiments show that temperature can affect the configuration of a cell or a synthetic membrane. (Because membranes expand more rapidly than their contens, higher temperatures favour vesicles.) The difference of osmotic and mechanical pressure across the surface should matter, although very little has been done with that.

In some real-life membranes, it may also be significant that some of the molecular components are linked laterally to others by covalent forces; a year ago, B. Fourcade from the Institut Laue-Langevin at Grenoble and M. Mutz and D. Bensimon from the Ecole Normale Supérieure in Paris used a synthetic membrane whose components were sideways polymerizable to recover toroidal vesicles closely resembling erythrocytes (Phys. Rev. Lett. 68, 2,551; 1992).

A further complication is that the quantity that truly determines the shape of a membrane surface is the free energy, which depends on entropy as well as energy. People are busily measuring the fluctuations of membrane surfaces to make some estimate of how important that may be. Horrendous problems in topology and the variational calculus arise if people are not careful.

Another assumption to be relaxed is that the chemical composition of a membrane is uniform, which is plainly far from the truth. One of the functions of the cell membrane, after all, is to carry the receptors and other proteins that allow a cell to interact with others. This topic has been enlivened in the past few years by the discovery that, in membranes made of mixtures of phospholipids and cholesterol, two separate phases, both of them fluid, can be seen to separate. One is rich in phospholipids, the other in cholesterol.

Two complementary aspects of bilayer membranes with more than one component have now been explored. A few weeks ago, Udo Seifert from the Solid-State Physics Institute at Jülich in Germany showed how the energetics of the problem would allow embryo vesicle buds, perhaps induced by temperature change, to induce inhomogeneities of membrane composition, perhaps forming patches of similar membrane molecules around a nucleating centre.

Now Frank Jülicher and Reinhard Lipowsky (see above) from the same institute have turned the problem around, and have shown that if there is some degree of phase separation on the membrane, the interaction energy along the contour separating the two domains may be just enough to pinch off the membrane carrying one of the phases as a separate vesicle. It is remarkable that there are experimental data to show this to be the case.

None of this implies that it will soon be possible to calculate the production of vesicles carrying, say, neurostransmitter molecules from the synapses of neurons. But it is something to be proud of that the outlines of the physics of the problem have been clarified. What seems most needed now is the injection of a little real-life chemistry into an intriguing line of enquiry.

John Maddox 